\title{
Lattice Boltzmann Multicomponent Model for Direct-Writing Printing
}

\author{
Michele Monteferrante, ${ }^{1,}$ a) Andrea Montessori, ${ }^{1}$ Sauro Succi, ${ }^{1,2,3}$ Dario Pisignano, ${ }^{4,5}$ and Marco Lauricella ${ }^{1, b)}$ \\ ${ }^{1)}$ Istituto per le Applicazioni del Calcolo CNR, Via dei Taurini 19, 00185 Rome, \\ Italy \\ ${ }^{2)}$ Center for Life Nano Science@La Sapienza, Istituto Italiano di Tecnologia, 00161 Roma, \\ Italy \\ 3) Harvard Institute for Applied Computational Science, Cambridge, Massachusetts, \\ United States \\ 4) Dipartimento di Fisica, Università di Pisa, Largo B. Pontecorvo 16 3, 56127 Pisa, \\ Italy \\ 5) NEST, Istituto Nanoscienze-CNR, Piazza S. Silvestro 12, 56127 Pisa, Italy
}

We introduce a mesoscale approach for the simulation of multicomponent flows to model the direct-writing printing process, along with the early stage of ink deposition. As an application scenario, alginate solutions at different concentrations are numerically investigated alongside processing parameters, such as apparent viscosity, extrusion rate, and print head velocity. The present approach offers useful insights on the ink rheological effects upon printed products, susceptible to geometric accuracy and shear stress, by manufacturing processes such as the direct-writing printing for complex photonic circuitry, bio-scaffold fabrication, and tissue engineering.

\section{INTRODUCTION}

In the last decade, 3D printing processes have gained enormous attention as tool for additive manufacturing in many fields of science and engineering. The major success of 3D printing is mostly due to the digital process control, which offers remarkable flexibility in terms of patterning through material deposition. By exploiting a computer-controlled layer-by-layer fabrication technique, soft materials are utilised in fused deposition modelling and in extrusion directwriting bio-plotters that apply a pressure gradient to fluids, possibly generating architected matter with qualitatively new properties 12 . As a consequence, this set of technologies is nowadays exploited in a wide variety of applications, such as tissue engineering (e.g., bio-compatible scaffolds), microsystems (lab-on-chip), microelectronics (sensors), and aerospace structures (aircraft engine bracket), to name a few ${ }^{3 / 5}$. The vast potential of additive manufacturing requires, however, an unprecedented control over several aspects of the soft materials involved in the 3D printing process. Their dynamics, composition, structure, function and rheology are all key elements, which severely affect the features of the finally produced parts and structures.

In this framework, efficient numerical simulations might offer a crucial help to understand the relevant, and interplaying, characteristics of the fluids and experimental setups, similarly to other manufacturing processes where computational tools have been successfully applied in the last two decades (e.g. electrospinning ${ }^{6}$, electrospray ${ }^{7}$ ). In the direct-writing printing context, numerical simulations could be used to maximise the printability of a given ink, avoid process failures and anticipate the microstructural properties of the products, by providing a set of observables (e.g., flow rate, stress tensor), which are often difficult to access experimentally. Printability is usually studied empirically, and a priori criteria are not available that, given an ink and a prototype model, allow the success of the manufacturing process to be reliably predicted. Moreover, the challenging characteristic length scale of the process, i.e. the diameter of the print head nozzle used to deposit materials, significantly constrain the possible choices within available computational methods. For instance, microscopic techniques, such as molecular dynamics, are generally unable to access length and time scales of experimental relevance, for want of computing resources. On the other hand, finite volume or finite element methods may also become computationally expensive in the presence of moving boundary conditions, such as the ones describing the moving print head.

Mesoscale techniques, and particularly the Lattice Boltzmann (LB) method ${ }^{8} 10$ offer an appealing alternative to both methods above, eventually striking an optimal balance between the two. Indeed LB moves noise-free discrete probability distributions along force-free (straight) trajectories and represents the effect of molecular collisions through the relaxation towards a suitable lattice local-equilibrium. Once the lattice symmetry and the local equilibria are suitably designed, the scheme can be shown to reproduce quantitatively the Navier-Stokes equations of fluid flows. The result is a very elegant and efficient computational scheme, featuring outstanding amenability to parallel implementations also in the presence of strong nonlinearities and complex boundary conditions ${ }^{8|9| 11]}$.

\footnotetext{
a) First author

b) Corresponding author: m.lauricella@iac.cnr.it
} 
In this work, we open a new route for predicting 3D printability, developing the regularised version of the Colour Gradient (CG) Lattice Boltzmann (LB) model 10 to account for the non-Newtonian rheological behaviour, typical of $3 \mathrm{D}$ printed pseudo-plastic inks. These systems endure a largely varying apparent viscosity, depending on the shear rate 12 . The regularised version of the LB method mitigates issues related to both low and high viscosities ${ }^{13}$, the first threatening numerical stability, while the latter undermining the very hydrodynamic limit of the LB scheme. Further, the shift in the nozzle position during actual 3D printing processes is also included. As a practical application, we focus on sodium alginate solutions, which are widely used in direct-writing printing to manufacture scaffolds for cell cultures and tissue regeneration ${ }^{4}$. The printing accuracy is discussed in terms of a Parameter Optimization Index $(\mathrm{POI}) \sqrt{14}$, which is predicted in terms of the numerical inputs.

\section{MODEL DETAILS}

\section{A. Regularized colour-gradient lattice Boltzmann model}

The regularized CG method for multicomponent-multiphase systems provides computationally efficient access to capillary number regimes relevant to microfluidics 15 .

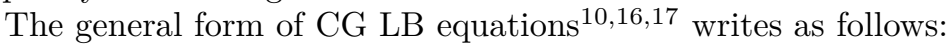

$$
f_{i}^{k}\left(\vec{x}+\vec{c}_{i} \Delta t, t+\Delta t\right)=f_{i}^{k}(\vec{x}, t)+\Omega_{i}^{k}\left[f_{i}^{k}(\vec{x}, t)\right]
$$

where $k$ is the colour component, $i$ is the index spans the lattice discrete directions, and $\Omega_{i}^{k}$ denotes the collision operator of the Colour Gradient model. In Eq. 1. $f_{i}^{k}$, represents the probability of finding a particle of the $k-t h$ component at position $\vec{x}$ and time $t$ with discrete lattice velocity $\vec{c}_{i}$. For the sake of simplicity, we adopt the standard D2Q9 lattice, and only two colours are assumed in the system, i.e. yellow and blue, standing for the dense ink and air, respectively. In the following, $i=0$ denotes the resting population with zero velocity, while $i \in[1, \ldots, 8]$ represent the directions at angle $\theta=(i-1) \pi / 4$ with velocity modulus $\left|\vec{c}_{i}\right|=1$ for $i \in[1,3,5,7]$, and $\left|\vec{c}_{i}\right|=\sqrt{2}$ for $i \in[2,4,6,8]$ in lattice units, assumed $\Delta x_{\mathrm{LB}}=1$ and $\Delta t_{\mathrm{LB}}=1$. A comparison among the regularized version of the Colour Gradient (CG) lattice Boltzmann (LB) mode 15 and other LB diffuse interface approaches (e.g., pseudo-potential model, free energy model) was discussed by S. Leclaire and coworkers ${ }^{18}$ noting that, although both pseudo-potential and CG methods are able to reproduce the correct physics, macroscopic parameters such as surface tension and interface thickness can be set independently only in CG method, while pseudo-potential model needs a posteriori adjustment of the repulsive forces between different species to match the required physics. The density, $\rho_{k}$, of the $k-t h$ fluid component is assessed as the zeroth moment of the distribution functions

$$
\rho^{k}(\vec{x}, t)=\sum_{i} f_{i}^{k}(\vec{x}, t)
$$

while the total momentum, $\rho \vec{u}$, is defined by the first order moment:

$$
\rho \vec{u}=\sum_{k} \sum_{i} f_{i}^{k}(\vec{x}, t) \vec{c}_{i},
$$

being $\rho$ the sum of the two component densities. The collision operator, $\Omega_{i}^{k}$, results from the combination of three sub-operators:

$$
\Omega_{i}^{k}=\left(\Omega_{i}^{k}\right)^{(3)}\left[\left(\Omega_{i}^{k}\right)^{(1)}+\left(\Omega_{i}^{k}\right)^{(2)}\right]
$$

The first, $\left(\Omega_{i}^{k}\right)^{(1)}$, is the standard BGK operator:

$$
\left(\Omega_{i}^{k}\right)^{(1)}=-\frac{1}{\tau}\left[f_{i}^{k}(\vec{x}, t)-f_{i}^{k, e q}(\vec{x}, t)\right]
$$

where $\tau$ is a relaxation time setting the numerical viscosity of the mixture (see below) and $f_{i}^{k, e q}(\vec{x})$ is a modified equilibrium distribution function:

$$
\begin{aligned}
& f_{i}^{k, e q}\left(\vec{x}, \rho_{k}, \vec{u}\right)= \\
& \rho^{k}\left(\phi_{i}^{k}+w_{i}\left[\frac{\vec{c}_{i} \cdot \vec{u}}{c_{s}^{2}}+\frac{\left(\vec{c}_{i} \cdot \vec{u}\right)^{2}}{2 c_{s}^{2}}-\frac{(\vec{u})^{2}}{2 c_{s}^{2}}\right]\right),
\end{aligned}
$$


with $c_{s}$ the lattice sound speed and $w_{i}$ the weights of the standard D2Q9 lattice ${ }^{10}$ : $w_{0}=4 / 9, w_{1,3,5,7}=1 / 9$, $w_{2,4,6,8}=1 / 36$. Here, the coefficients, $\phi_{i}^{k}$, read ${ }^{17}$.

$$
\phi_{i}^{k}= \begin{cases}\alpha_{k}, & i=0, \\ \left(1-\alpha_{k}\right) / 5, & i=1,3,5,7, \\ \left(1-\alpha_{k}\right) / 20, & i=2,4,6,8,\end{cases}
$$

and are tuned to simulate systems with different density ratio $\gamma$ :

$$
\gamma=\frac{\rho_{Y}}{\rho_{B}}=\frac{1-\alpha_{B}}{1-\alpha_{Y}}
$$

with the apexes $Y$ and $B$ standing for yellow and blue fluid component, respectively. The partial pressure of $k$-th component reads:

$$
p^{k}=\frac{3}{5} \rho^{k}\left(1-\phi_{0}^{k}\right)
$$

The second operator, $\left(\Omega_{i}^{k}\right)^{(2)}$, called perturbation operator, generates the interfacial tension and has the form:

$$
\left(\Omega_{i}^{k}\right)^{(2)}=\frac{A}{2}\left|\nabla \rho^{N}\right|\left[w_{i} \frac{\left(\vec{F}_{\mathrm{cg}} \cdot \vec{c}_{i}\right)^{2}}{\left|\vec{F}_{\mathrm{cg}}\right|^{2}}-B_{i}\right],
$$

where $\vec{F}_{\text {cg }}$ denotes the colour gradient force, reading:

$$
\vec{F}_{\mathrm{cg}}=\frac{\rho_{B}}{\rho} \nabla\left(\frac{\rho_{Y}}{\rho}\right)-\frac{\rho_{Y}}{\rho} \nabla\left(\frac{\rho_{B}}{\rho}\right) .
$$

As observed in Refs $\frac{19}{21}$, the gradient for an arbitrary observable $\chi$ can be obtained by the second-order isotropic central scheme :

$$
\nabla \chi(\vec{x}, t)=\frac{1}{c_{s}^{2}} \sum_{i} w_{i} \chi\left(\vec{x}+\vec{c}_{i}, t\right) \vec{c}_{i}
$$

In Eq. 10 the $B_{i}$ coefficients depend on the lattice (taken: $B_{0}=-4 / 27, B_{1.3 .5 .7}=2 / 27, B_{2,4,6,8}=5 / 108$ from Ref:[17), whereas $A$ is a free parameter modeling the surface tension, $\sigma$, that is.:[17|22].

$$
\sigma=\frac{2 \tau}{9} A,
$$

where $\tau$ is the effective relaxation time. The recoloring operator $\left(\Omega_{i}^{k}\right)^{(3)}$ is necessary since the perturbation operator alone does not guarantee the phase separation:

$$
\begin{array}{r}
\left(\Omega_{i}^{Y}\right)^{(3)}=\frac{\rho_{Y}}{\rho} f_{i}^{*}+\beta \frac{\rho_{Y} \rho_{B}}{\rho^{2}} \cos \left(\varphi_{i}\right) \sum_{k} f_{i}^{e q}\left(\vec{x}, \rho_{k}, \vec{u}=0\right) \\
\left(\Omega_{i}^{B}\right)^{(3)}=\frac{\rho_{B}}{\rho} f_{i}^{*}-\beta \frac{\rho_{Y} \rho_{B}}{\rho^{2}} \cos \left(\varphi_{i}\right) \sum_{k} f_{i}^{e q}\left(\vec{x}, \rho_{k}, \vec{u}\right)
\end{array}
$$

Here, $\beta$ is a parameter tuning the thickness of the diffuse interface, $f_{i}^{*}$ is the post collision total density in the lattice direction $i, f_{i}^{e q}=\sum_{k} f_{i}^{k, e q}$ and finally:

$$
\cos \left(\varphi_{i}\right)=\frac{\vec{c}_{i} \cdot \nabla \rho_{N}}{\left|\vec{c}_{i}\right|\left|\nabla \rho_{N}\right|} .
$$

The kinematic viscosity, $\nu$, is assessed as $\frac{16 \mid 17}{\text { : }}$

$$
\frac{1}{\nu}=\frac{\rho_{Y}}{\rho} \frac{1}{\nu_{Y}}+\frac{\rho_{B}}{\rho} \frac{1}{\nu_{B}},
$$

being $\nu_{Y}$ and $\nu_{B}$ the density of the yellow and blue component, respectively. In order to model the wettability on the different walls in the system, see Fig. 1 and compute the gradients of $\rho^{k}$ by Eq. 12 also close to the boundaries, we 
set a fictitious fluid density, $\rho_{s}^{k}$, for the two components on all the wall nodes 23 . The fictitious densities are estimated by the extrapolation of the color function at neighboring fluid lattice nodes by the formula:

$$
\rho_{s}^{k}(\vec{x}, t)=\zeta^{k}(\vec{x}, t) \frac{\sum_{i} w_{i} \rho^{k}\left(\vec{x}+\vec{c}_{i}, t\right)}{\sum_{i} w_{i}} s\left(\vec{x}+\vec{c}_{i}, t\right),
$$

where $\zeta^{k}(\vec{x}, t)$ is a positive parameter tuning the affinity of the different walls, see Fig. 1 at the position $\vec{x}$ for a given fluid component, and $s$ is a switch function taking value one if the site at $\vec{x}+\vec{c}_{i}$ is a fluid and is zero otherwise. The contact angle is given by:

$$
\theta=\arccos \left(\frac{\rho_{s B}-\rho_{s Y}}{\rho_{B}^{0}}\right)
$$

where $\rho_{B}^{0}$ is the initial density of the blue fluid. Note the present strategy can be interpreted as a simplified version of the approaches reported in Refs ${ }^{2425}$ where the wall densities are interpolated with the same expression of Eq. 18 without the $\zeta^{k}$ but the density's gradient is subsequently rotated to match the prescribed contact angle. Although less accurate in reproducing the contact angle for the presence of spurious currents, our approach is a simple procedure to model hydrophobicity $\left(\zeta^{k}<1\right)$ or hydrophilicity $\left(\zeta^{k}>1\right)$ of the walls as given in Fig. 1

Implying the Einstein convention for summation over Greek indices (see Appendix A of Ref. ${ }^{9}$ ), the regularization step reads 15 .

$$
f_{i}^{k, r e g}(\vec{x}, t)=f_{i}^{k, e q}\left(\vec{x}, \rho_{k}, \mathbf{u}\right)+\frac{w_{i}}{2 c_{s}^{4}} Q_{i \alpha \beta} \Pi_{\alpha \beta}^{n e q, k}
$$

where $Q_{i \alpha \beta}=\left(c_{i \alpha} c_{i \beta}-c_{s}^{2} \delta_{\alpha \beta}\right)$ and $\Pi_{\alpha \beta}^{n e q, k}=\left(\sum_{i} f_{i}^{k} c_{i \alpha} c_{i \beta}\right)-\left(\sum_{i} f_{i}^{k, e q} c_{i \alpha} c_{i \beta}\right)$ with $\alpha, \beta$ denoting Cartesian directions and $\delta$ the Kronecker delta. Note that Eq. 20 consists of a projection of a distribution functions, $f_{i}^{k}$, onto the set of Hermite basis. In doing so, we obtain a set of filtered distribution functions, $f_{i}^{k, r e g}$, which depends only on the first and second macroscopic hydrodynamic moments without higher-order non-equilibrium information often referred as ghost moments $\frac{13|15| 26}{\text {. It was shown }}{ }^{27 \mid 29]}$ that the procedure provides general benefits in terms stability in the BGK LB scheme, which can be decisive in the case of low-viscosity simulations. Hence, the regularized distributions, $f_{i}^{k, r e g}$ are used in Eq. 1

The hydrodynamic limit of Eq. $1^{10 \mid 22}$ is found to converge to a set of equations for the conservation of mass and linear momentum:

$$
\begin{array}{r}
\frac{\partial \rho}{\partial t}+\nabla \cdot \rho \vec{u}=0 \\
\frac{\partial \rho \vec{u}}{\partial t}+\nabla \cdot \rho \vec{u} \vec{u}=-\nabla p+\nabla \cdot\left[\rho \nu\left(\nabla \vec{u}+\nabla \vec{u}^{T}\right)\right]+ \\
+\nabla \cdot \Sigma
\end{array}
$$

where $p=\sum_{k} p_{k}$ is the pressure, $\nu=c_{s}^{2}(\tau-1 / 2)$ is the kinematic viscosity of the mixture, $\boldsymbol{\Sigma}=-\tau \sum_{k} \sum_{i}\left(\Omega_{i}^{k}\right)^{(2)} \vec{c}_{i} \vec{c}_{i}$ is the stress tensor of the curved interface, and $\tau$ is a time tuning the relaxation of the fluid flow towards its local equilibrium used in the collision operator, $\left(\Omega_{i}^{k}\right)^{(1)}$, of Eq. 5 . At each time step before the collision in Eq. 1 , all the populations, $f_{i}^{k}$, are filtered by applying the regularization step $13[15$.

It is worth to highlight that the LB approach avoids two potential and serious issues of computational physics in discretizing the Navier-Stokes equations of continuum fluid mechanics: strong non-linearity and complex geometry within a time-dependent formulation. In particular, the discretization of the non-linear term, $\nabla \cdot \rho \vec{u} \vec{u}$, in the NavierStokes equations requires the non-locally derivative approximations over the adjacent space in numerical techniques such as finite-difference methods and finite element methods. In contrast, the LB method disentangles the non-locality and non-linearity of the problem, since the non-linearity is treated locally (collision step of Eq. 1), and the non-locality is treated linearly (streaming step of Eq. 1) as a shift in memory over the adjacent nodes. Thus, it turns out that the LB approach is a very attractive computational bargain to high-performance computing on parallel architectures, including GPUs 89 .

\section{B. Extension to non-Newtonian flow and moving print head}

To model the non-Newtonian fluids, the model is extended in similarity with the approach reported in Refs 30 33. The extension consists essentially of determining the local value of the relaxation time, in such a way that the desired 
local value of the viscosity is recovered to match a constitutive equation for the stress tensor 32 35. We assume that the shear-thinning model introduced originally by M. Cross ${ }^{12}$ (in the following referred to as Cross model) adequately describes the ink viscosity. Note that the Cross model was already employed to describe the non-Newtonian behavior of alginate solutions by Roopa and Bhattacharya ${ }^{36}$. However, other possible models can be freely adopted without losing the applicability of the present approach. The Cross model states:

$$
\mu(\dot{\gamma})=\mu_{\infty}+\frac{\mu_{0}-\mu_{\infty}}{1+(\lambda \dot{\gamma})^{n}}
$$

where $\mu$ is the dynamic viscosity, $n$ the flow index ( $n<1$ for a pseudoplastic fluid), $\mu_{0}$ the zero shear viscosity, $\mu_{\infty}$ the asymptotic value, and $\lambda$ the retardation time at which the shear-thinning starts. In the following, the yellow dense component (the ink) is assumed to show a non-Newtonian behaviour ${ }^{12}$ while the blue component (the air) is a Newtonian fluid. Far from the interface, the stress tensor and the strain tensor are mainly represented by the k-th component so that $\Pi_{\alpha \beta} \sim \Pi_{\alpha \beta}^{k}$ and $\Gamma_{\alpha \beta} \sim \Gamma_{\alpha \beta}^{k}$, respectively. Following the literature ${ }^{8[35}$, the stress tensor $\Pi_{\alpha \beta}$ relates with the strain tensor $\Gamma_{\alpha \beta}$ by the relation $\Gamma_{\alpha \beta}=-\left(1 / 2 \rho \tau c_{s}^{2}\right) \Pi_{\alpha \beta}$, where the stress tensor $\Pi_{\alpha \beta}=\sum_{i}\left(f_{i}-f_{i}^{e q}\right) \vec{c}_{i \alpha} \vec{c}_{i \beta}$. Thus, in the yellow fluid bulk the last relation can be rewritten as:

$$
\dot{\gamma}_{Y}\left(\left|\Pi_{\alpha \beta}^{Y}\right|\right)=\frac{\left|\Pi_{\alpha \beta}^{Y}\right|}{\rho_{Y} \tau_{Y}\left(\dot{\gamma}_{Y}\right) c_{s}^{2}}
$$

where the stress tensor of the yellow component reads $\Pi_{\alpha \beta}^{Y}=\sum_{i}\left(f_{i}^{Y}-f_{i}^{Y, e q}\right) \vec{c}_{i \alpha} \vec{c}_{i \beta}$, and the tensor norms are computed as $\dot{\gamma}_{Y}=2\left|\Gamma_{\alpha \beta}^{Y}\right|=2 \sqrt{\sum_{\alpha \beta} \Gamma_{\alpha \beta}^{Y} \Gamma_{\alpha \beta}^{Y}}$ and $\left|\Pi_{\alpha \beta}^{Y}\right|=\sqrt{\sum \Pi_{\alpha \beta}^{Y} \Pi_{\alpha \beta}^{Y}}$ with the relaxation parameter $\tau_{Y}$ setting the kinematic viscosity of the yellow fluid, $\nu_{Y}=c_{s}^{2}\left(\tau_{Y}-1 / 2\right)$.

Since $\mu(\dot{\gamma})=\rho \nu(\dot{\gamma})$ and $\mu(\dot{\gamma})=\rho c_{s}^{2}(\tau(\dot{\gamma})-1 / 2)$, the function $\tau_{Y}\left(\dot{\gamma}_{y}\right)$, requested in Eq. 24, can be obtained by Eq. 23 as:

$$
\tau_{Y}\left(\dot{\gamma}_{Y}\right)=\frac{1}{2}+\frac{1}{c_{s}^{2}}\left[\nu_{\infty, Y}+\frac{\nu_{0, Y}-\nu_{\infty, Y}}{1+\left(\lambda \dot{\gamma}_{Y}\right)^{n}}\right]
$$

Inserting Eq. 25 in Eq. 24 provides an implicit equation in the variable $\dot{\gamma}_{Y}$, which is solved iteratively, performing several iterations as long as the value of $\dot{\gamma}_{Y}$ is not converged below a given threshold. If close to the interface, $\tau$ is computed from the interpolated value of the viscosity by Eq. 17. A similar approach was exploited by Pontrelli et al ${ }^{31}$ to model a pseudo-plastic single-phase fluid, and it was validated by comparison with the analytical solution of Poiseuille flow with the power-law model.

Since the print head moves during the process, we needed a particular treatment of the boundary conditions of the nozzle walls and fluid nodes around the nozzle. Inspired to the trailblazing work by Antony Ladd 37 , we define a template of solid nodes with an internal reference system, which is translated along the time evolution by integrating an equation of motion. In accordance with the formulation proposed by F. Jansen and J. Harting 3 , only the exterior regions are filled with fluid, whereas the interior parts of the nozzle is considered solid nodes.

Denoting with $f_{i}^{*}\left(\vec{x}_{b}, t\right)$ the post collisional distribution at the boundary position $\vec{x}_{b}$ hitting the nozzle wall in the direction $\vec{c}_{i}$ and located in the middle position along the link connecting the solid node $\vec{x}_{s}$ from the boundary fluid node $\vec{x}_{b}$, we exploit a simple generalization of the halfway bounce-back rule ${ }^{9 / 37 / 39}$. Hence, the streaming step proceeds as:

$$
f_{\bar{i}}^{k}\left(\vec{x}_{b}, t+1\right)=f_{i}^{k}\left(\vec{x}_{b}, t\right)-2 w_{i} \rho_{w}^{k} \frac{\vec{v}_{\text {nozzle }} \cdot \vec{c}_{i}}{c_{s}^{2}}
$$

where $\bar{i}$ is the lattice direction $-\vec{c}_{i}$. The symbol $\rho_{w}^{k}$ in Eq. 26 denotes the density at the wall position, which is obtained by a third order interpolation in the direction $-\vec{c}_{i}$.

Because the print head moves over the lattice nodes, it happens that a subset of fluid boundary nodes in front of the moving nozzle crosses its surface, becoming solid nodes. Similarly, a subset of interior nodes on the surface is released at the back of the nozzle. The two distinct events require the destruction and the creation of fluid nodes, respectively. Following the previous strategy reported in the literature, whenever a fluid node changes to solid, the fluid is deleted ${ }^{40}$ without transferring its linear momentum to the nozzle beneath the nozzle infinite mass hypothesis. In the creation fluid node step, as the nozzle leaves a lattice site, new fluid populations are initialized from the equilibrium distributions, $f_{i}^{e q, k}\left(\bar{\rho}^{k}, \vec{v}_{\text {nozzle }}\right)$, for the two $k$-th components with the velocity of the nozzle wall, $\vec{v}_{\text {nozzle }}$ and the $k$-th fluid density taken as its average value, $\bar{\rho}^{k}$, computed over the neighbouring fluid nodes $38 \mid 40$.

As a first approximation, the solid-fluid interactions are accounted only for the part concerning the effect of the moving nozzle on the surrounding fluid and not viceversa, which is equivalent to assume that the motion of the print 
head is fully controlled by the digital system of the 3D printer (nozzle infinite mass hypothesis). Hence, a constant velocity $\vec{v}_{\text {nozzle }}$ of the nozzle (print head) is taken as an input parameter to describe the linear motion of the nozzle, and a drag force is added close to the deposition zone in order to model the friction between the ink and the collector.

Inside the nozzle, the yellow component (the ink) is inserted with constant velocity $\vec{u}_{\text {ink }}$ with respect to the internal reference frame on the print head. Considering that the nozzle reference frame is moving with $\vec{v}_{\text {nozzle }}$, the total fluid velocity inserted at the inlet reads:

$$
\vec{u}_{\text {Inlet }}=\vec{u}_{\text {ink }}+\vec{v}_{\text {nozzle }}
$$

Hence, $\vec{u}_{\text {Inlet }}$ replaces $\vec{v}_{\text {nozzle }}$ in Eq. 26, which is used to model the fluid inlet inside the print head.

We also used a Dirichlet boundary conditions in our work to maintain the pressure (density) of the blue fluid (air) constant. For the Dirichlet condition, the anti-bounce back scheme ${ }^{41}$ is used instead for constant pressure (densities) boundaries:

$$
\begin{array}{r}
f_{\bar{i}}^{k}\left(\vec{x}_{b}, t+1\right)=-f_{i}^{k *}\left(\vec{x}_{b}, t\right)+ \\
+2 \rho_{w}^{k}\left\{\phi_{i}^{k}+w_{i}\left[\frac{\left(\vec{c}_{i} \cdot \vec{u}_{w}\right)^{2}}{2 c_{s}^{4}}-\frac{\vec{u}_{w}^{2}}{2 c_{s}^{2}}\right]\right\},
\end{array}
$$

where $\rho_{w}^{k}$ is the imposed density at the open boundary and $\vec{u}_{w}$ is the velocity at half-way point estimated by a second order interpolation along the direction $-\vec{c}_{i}$.

The drag force modelling the friction between the ink and the collector reads:

$$
F_{d}(\vec{x}, t)=-\gamma \rho_{Y}(\vec{x}, t) \vec{u}(\vec{x}, t),
$$

where $\gamma$ is the friction coefficient tuning the drag force. This force is turned on at grid points which are closer than 4 lattice units from the deposition wall. The drag force is added in Eq. 5 by the exact difference method proposed by Kupershtokh and coworkers $\stackrel{42}{ }$.

\section{SYSTEM SETUP}

It is worth to remark the main simplifying assumptions adopted in the simulations. In the present paper, the Cross model is adopted to describe the non-Newtonian fluid, albeit any other rheological relation can be adopted, obviously in the context of pseudo-plastic models (e.g., Carreau Model, Herschel-Bulkley model, etc.). Further, the adhesion property of the fluid onto the deposition surface can be relevant. The contact angle is set equal to $90^{\circ}$, corresponding to the neutral affinity of the ink to the surface (neither hydrophilic nor hydrophobic). Nonetheless, different contact angle values can be investigated by the present model. Finally, we exploit a two-dimensional description of the system to reduce both the computational cost and the wall-clock time necessary for each simulation. As a consequence, the lateral shear effect of the slender fluid filament along the third dimension is neglected in the two-dimensional description. Nonetheless, the comparison with experimental data in Section [V] will show as the two-dimensional approximation does not invalidate the agreement between the numerical results and the experimental counterpart. In other words, the validation of the present model is not prevented from the dimension reduction.

The alginate concentration in water is taken in the range $1 \%-3 \% \mathrm{w} / \mathrm{v}$, allowing the investigation of the fluid characteristics for optimal printing. Alginate solutions are shear-thinning non-Newtonian fluids $36 / 43$. Thus, the viscosity decreases to smaller value as the shear rate increases. This dependence of the viscosity on the shear rate makes the whole printing process complex, since on the one side, low viscosity reduces shear forces, thus speeding up printing, but it can also reduce both resolution and accuracy 14 . The dynamic viscosity $(0.2-4.6 \mathrm{~Pa} \cdot \mathrm{s})$ observed in alginate solutions represents a good compromise between the above criteria 36 . Further, it is worth underlining that solutions with higher alginate concentrations (up to 9\%) have been recently used for bioscaffold fabrication ${ }^{44}$ with dynamic viscosity values falling in a narrow window $(2.4-2.7 \mathrm{~Pa} \cdot \mathrm{s})$ of the range considered in the present work. Thus, the present model is reasonable able to probe also alginate solution at higher concentration values. On the other hand, in the actual work we focus the attention on the alginate concentrations in the range $1 \%-3 \% \mathrm{w} / \mathrm{v}$ alongside with the corresponding parameters of the Cross model reported by Roopa and Bhattacharya ${ }^{36}$. Sarker and Chen 43 have also investigated the rheology of the alginate solutions, although some parameters as $\lambda$ and $n$ were not explicitly given. The kinematic viscosity of the air is set $\nu_{\text {air }}=1.552 \cdot 10^{-5} \mathrm{~m}^{2} / \mathrm{s}$, corresponding to the kinematic viscosity at $25{ }^{\circ} \mathrm{C}$. The rheological parameters of the ink fluid and the air are reported in Tab. I To model the fluid-air system we simulate two fluids with a density ratio $\gamma=842.0\left(\approx\right.$ the water/air density ratio at $\left.25^{\circ} \mathrm{C}\right)$, while the surface

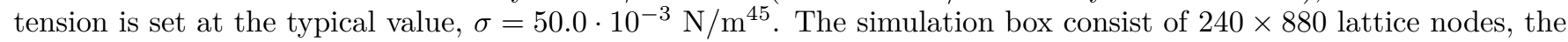
nozzle diameter of the channels was fixed at $d=60$ lattice nodes, and the same value was assigned to the distance 
of the nozzle by the deposition surface corresponding to 60 lattice nodes. This configuration is characteristic of $3 \mathrm{D}$ bio-plotters 46 . The system is initialised with the nozzle filled up with ink and located on the right side of Fig. 1 , that also shows graphically the various boundary conditions used in the simulations. These were set periodic along the $x$-axis, while along the $y$ - axis, the bottom side is a no-slip wall and the top boundary outside the nozzle is set to a constant blue (air) density $\rho^{B}$.

\begin{tabular}{l|c|c|c|c|c} 
& $\nu_{0}\left(\mathrm{~m}^{2} \mathrm{~s}\right)$ & $\nu_{\infty}\left(\mathrm{m}^{2} \mathrm{~s}\right)$ & $\lambda(\mathrm{s})$ & $\mathrm{n}$ & $\nu_{\text {air }}\left(\mathrm{m}^{2} \mathrm{~s}\right)$ \\
\hline $1 \%-25^{\circ} \mathrm{C}$ & $15.610^{-5}$ & $2.1110^{-5}$ & $3.1610^{-3}$ & 0.751 & $1.55210^{-5}$ \\
$2 \%-25^{\circ} \mathrm{C}$ & $66.710^{-5}$ & $0.6910^{-5}$ & $5.9610^{-3}$ & 0.713 & $1.55210^{-5}$ \\
$3 \%-25^{\circ} \mathrm{C}$ & $463.610^{-5}$ & $3.6410^{-5}$ & $62.510^{-3}$ & 0.573 & $1.55210^{-5}$ \\
$3 \%-40^{\circ} \mathrm{C}$ & $186.810^{-5}$ & $5.1410^{-5}$ & $10.210^{-3}$ & 0.737 & $1.55210^{-5}$ \\
\hline & $\nu_{0}(\mathrm{LB})$ & $\nu_{\infty}(\mathrm{LB})$ & $\lambda(\mathrm{LB})$ & $\mathrm{n}$ & $\nu_{\text {air }}(\mathrm{LB})$ \\
\hline $1 \%-25^{\circ} \mathrm{C}$ & 0.134 & 0.0181 & $3.31010^{-5}$ & 0.751 & $0.01 \overline{3}$ \\
$2 \%-25^{\circ} \mathrm{C}$ & 0.430 & 0.0045 & $8.32510^{-5}$ & 0.713 & 0.01 \\
$3 \%-25^{\circ} \mathrm{C}$ & 1.991 & 0.0156 & $131.010^{-5}$ & 0.573 & $0.00 \overline{6}$ \\
$3 \%-40^{\circ} \mathrm{C}$ & 0.802 & 0.0221 & $21.3710^{-5}$ & 0.737 & $0.00 \overline{6}$
\end{tabular}

Table I. Values of rheological parameters used in simulations. For the alginate solutions the parameters were given by Roopa and Bhattacharya ${ }^{36}$.

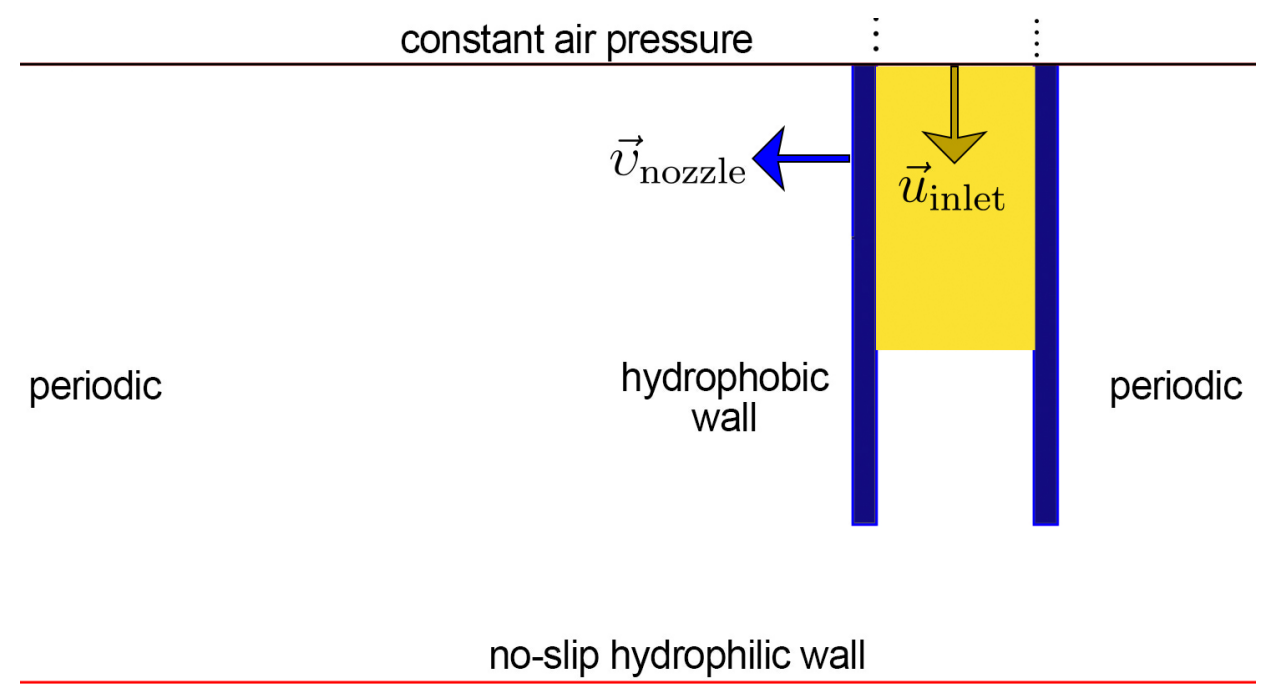

Figure 1. Representation of the system alongside with the different treatment of boundary conditions.

The ink velocity inside the nozzle in the internal reference frame is obtained from the mass flow rate given in literature ${ }^{43}$, by noting that:

$$
v_{\text {ink }}=\frac{\psi}{\rho \pi(d / 2)^{2}}
$$

As reported in $\frac{43}{}$, for a nozzle diameter of $0.2 \mathrm{~mm}$, typical value of flow rates $\psi$ are between $7.7 \mathrm{mg} / \mathrm{s}$ and $27 \mathrm{mg} / \mathrm{s}$.

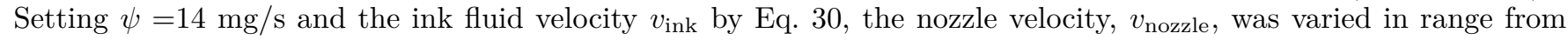
$0.25 v_{\text {ink }}$ to $1.75 v_{\text {ink }}$. Hence, $v_{\text {inlet }}$ is computed as: $\vec{u}_{\text {Inlet }}=\overrightarrow{\vec{v}}_{\text {ink }}+\vec{v}_{\text {nozzle }}$.

Denoting by the subscripts LB and MKS the physical observable in lattice and MKS system of units respectively, we adopted the following rules to convert the lattice units $\Delta x_{\mathrm{LB}}, \Delta t x_{\mathrm{LB}}, \Delta m_{\mathrm{LB}}$ in the corresponding physical quantities 
$\Delta x_{\mathrm{MKS}}, \Delta t x_{\mathrm{MKS}}, \Delta m_{\mathrm{MKS}}$. Assuming $\Delta x_{\mathrm{LB}}, \Delta t x_{\mathrm{LB}}, \Delta m_{\mathrm{LB}}$ equal to one, the lattice conversion rules are:

$$
\begin{array}{r}
\Delta x_{\mathrm{MKS}}=\frac{d_{\mathrm{MKS}}}{d_{\mathrm{LB}}}=3 . \overline{3} \cdot 10^{-6} \mathrm{~m} \\
\Delta m_{\mathrm{MKS}}=\frac{\left(\Delta x_{\mathrm{MKS}}\right)^{3} \rho_{\mathrm{MKS}}^{\text {air }}}{\rho_{\mathrm{LB}}^{B}}=4.39 \cdot 10^{-17} \mathrm{~kg} \\
\Delta t_{\mathrm{MKS}}=\frac{\left(\Delta x_{\mathrm{MKS}}\right)^{2} \nu_{\mathrm{LB}}^{B}}{\nu_{\mathrm{MKS}}^{B}}=[4.77-9.55] \cdot 10^{-9} \mathrm{~s}
\end{array}
$$

In particular, if we assume the nozzle diameter $d_{\mathrm{MKS}}=0.2 \cdot 10^{-3} \mathrm{~m}$ from Ref $\frac{43}{43}$ corresponding to $d_{\mathrm{LB}}=60$ lattice nodes, the $\Delta x_{\mathrm{MKS}}$ is determined by Eq. 31. while $\Delta m_{\mathrm{MKS}}$ is obtained by fixing the air density in lattice units $\rho_{\mathrm{LB}}^{B}$ equal to one. Since the kinematic viscosity of the air is always lower than the corresponding value in the ink, $\Delta t_{\mathrm{MKS}}$ is obtained by fixing $\tau^{B}$ and, thus, $\nu_{\mathrm{LB}}^{B}$ is also determined by the relation $\nu_{L B}^{B}=c_{s}^{2}\left(\tau^{B}-0.5\right)$, which is inserted in Eq. 33. In the following, $\tau_{\mathrm{LB}}^{B}$ and $\nu_{\mathrm{LB}}^{B}$ were fixed depending on the cases under investigations (see Tab. I ), so that $\Delta t_{\mathrm{MKS}}$ spans over the range reported in Eq. 33 However, $\tau^{B} \in[0.52, \ldots, 0.54]$ is taken sufficiently far from the limiting value 0.5 in order to avoid numerical instabilities 9 .

\section{RESULTS AND DISCUSSION}

In $3 \mathrm{D}$ printing, the ultimate printability of a given prototype depends both on the printer device and on the physical properties of the ink fluid. In order to assess the quality of the print process with respect to tunable parameters, we introduce a POI following 14: POI = accuracy/theoretical shear stress. In fact, it was found that the shear stress can be minimised by manipulating printing parameter ${ }^{14}$, since it is proportional to the inlet pressure $p$ and inversely proportional to the nozzle diameter $d$. Hence, assuming the accuracy to scale inversely with the thickness (height $h$ ) of a single printed thread, the POI is written as 14 :

$$
P O I \propto \frac{d}{h p}
$$

We include the coverage ratio (that is, the ratio of the sectional area of the ink thread, $A_{i}$, to the area of the rectangle circumscribing the thread, $A_{r}$, as in the middle panel of Fig. 2 a) in the definition of the accuracy and obtain:

$$
P O I \propto \frac{A_{i}}{A_{r}} \frac{d}{h \Delta p_{m}}
$$

where: we consider the shear stress as proportional to the variation of the pressure in the ink, that reaches a maximum pressure variation $\Delta p_{m}$ (variation with respect to the equilibrium pressure $p_{0}$ ) outside the nozzle at completion of the deposition process; $h$ is taken as the largest height value of the thread behind the moving nozzle.

Hence, all the POI values are normalised to a reference value, in order compare the results with the different parameters set. We take the reference POI as the largest value, $\max _{i} P O I_{i}$, corresponding to a perfect coverage ratio, $\left(A_{i} / A_{r}\right)=1$, across all the simulations.

As a result, the normalized POI reads: $P O I_{i}^{n o r m}=\frac{P O I_{i}}{\max _{i}\left\{P O I_{i}\right\}}$. Nonetheless, it is worth highlighting that the POI is here aimed to determine the process quality in the context of alginate-type inks used for manufacturing applications in the context, among others, of bio-scaffolds. Hence, the POI index involves the maximum pressure observed in the simulation to monitor the shear forces in the fluid. For other applications, such as manufacturing processes with polymeric inks, the shear stress can be relatively less important. Thus, other indexes of printing quality could be mainly focused on the geometrical precision rather than the shear forces in the fluid, for instance, in the contexts of nano-printing 47 or electrode fabrication 48149 .

For all the simulations, we stopped the run as the nozzle covers six time the nozzle diameter $d$ from the lattice position where the deposited ink first touches the collector, thus allowing the geometry of the printed thread to be completely developed in high-resolution printing $\frac{43}{\text {. }}$.

In top panel of Fig. $2 p$, a set of snapshots are reported, showing the ink mass densities map $\left(\rho^{Y}\right)$ at the end of the simulation for three different velocity conditions of the nozzle $v_{\text {nozzle }}=\{0.25,1.0,1.75\} v_{\text {ink }}$ and alginate solution with the lowest concentration $1 \%$. The shape of the deposited ink is found to be strongly dependent on $v_{\text {nozzle }}$, overflowing beyond the travelled length of six diameters for $v_{\text {nozzle }}=0.25 v_{\text {ink }}$ such to provide a poor printing quality. That a low dispensing speed compared to $v_{\text {ink }}$, providing a surplus of ink compared to the space spanned by the nozzle, would decrease the printing accuracy is in agreement with previous results, both experimental ${ }^{50151}$ and numerical 52 . 


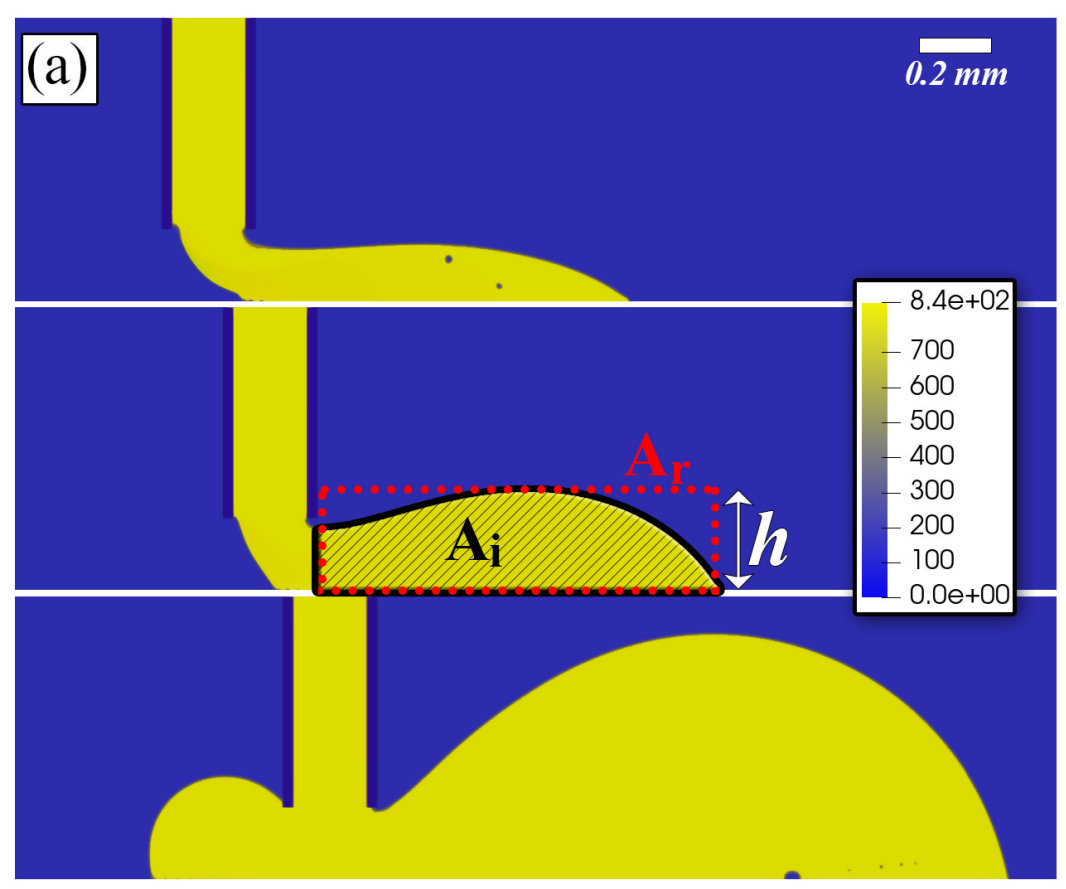

(b)

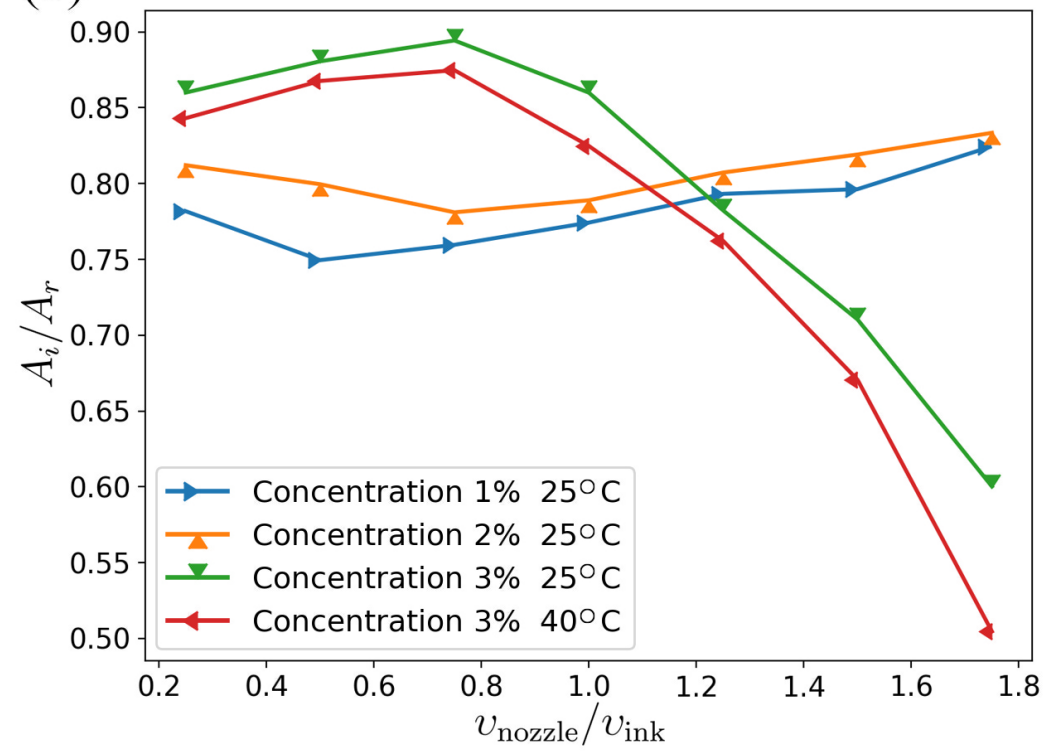

Figure 2. (a) Yellow fluid densities for $1 \%$ alginate solution. From top to bottom the nozzle velocities $v_{\text {nozzle }}$ are equal to $1.75 v_{\text {ink }}, 1.0 v_{\text {ink }}$, and $0.25 v_{\text {ink. }}$. The snapshots are taken as the nozzle has travelled six times, $6 d$, the nozzle diameter from the lattice position where the ink touches the deposition substrate. In the middle panel, we also sketch thread height $(h)$, the area of the ink thread $\left(A_{i}\right)$, and the area of the rectangle circumscribing the thread $\left(A_{r}\right)$ defining the coverage ratio, $A_{i} / A_{r}$.(b) Coverage ratio obtained by different printing parameters.

The height of the printed thread in Fig. 3a is also clearly dependent on the parameter $v_{\text {nozzle }}$. In order to study the dynamic evolution, we probe the maximum height, $h$, of the fluid thread behind the moving nozzle, investigating whether a stationary condition is reached along the deposition process.

The thread height, $h$, as a function of the distance travelled by the moving nozzle is reported in Fig. 3 In both the case of $1 \%$ and $3 \%$ concentrations, the height of the thread for lowest nozzle velocities, $v_{\text {nozzle }}=0.25 v_{\text {ink }}$ does not show any asymptotic trend, confirming that the printing precision is deteriorated by a low dispensing velocity. We also observe that the asymptotic values in $h$ decrease as the nozzle velocity increases for the $1 \%$ concentration, highlighting a clear, monotonic trend, which is in agreement with the experimental observations reported by Webb 

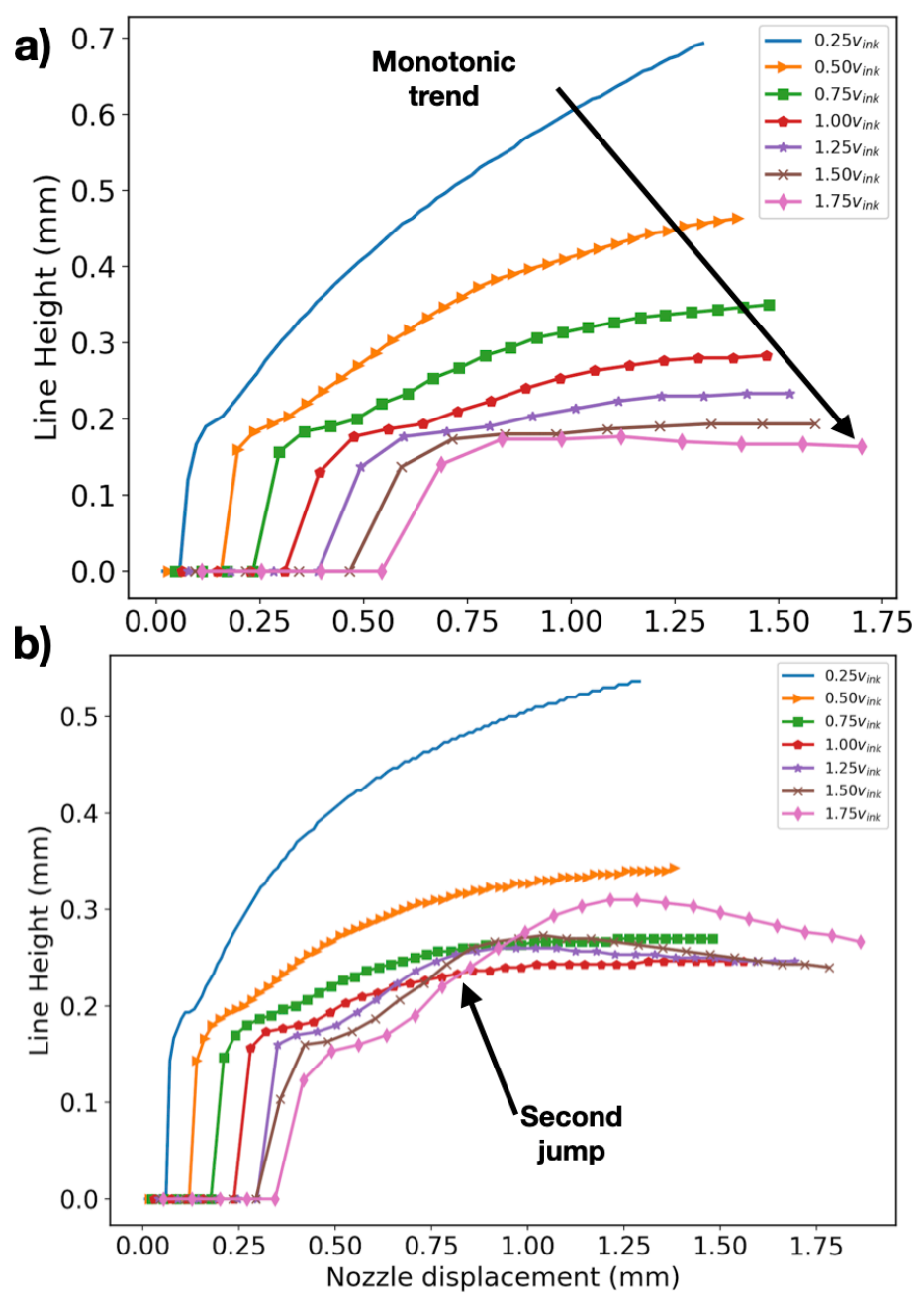

Figure 3. Thread height as a function of the nozzle displacement, for a $1 \%$ (top panel a) and $3 \%$ w/v (lower panel b) alginate solution. The temperature is equal to $25{ }^{\circ} \mathrm{C}$ in both the alginate solutions.

and Doyle 14 . It is worth to highlight as the numerical results trace qualitatively the experimental trend, although the model has been implemented in the two-dimensional framework, endorsing the validity of the dimension reduction.

The height evolution at high velocity and concentration $3 \% \mathrm{w} / \mathrm{v}$ shows a second jump, namely the thickness obtained at higher values of $v_{\text {nozzle }}$ overcomes the value measured with lower velocities $v_{\text {nozzle }}=[0.75,1.00,1.25] v_{\text {ink }}$, providing a non monotonic trend. This suggests that, at least at high ink concentration, an optimum operating value exists for the dispensing velocity, compared to the ink delivery rate, which minimises the thread height. Further, the sequence of asymptotes is found to be monotonic also in the case of $2 \%$ solutions at $25{ }^{\circ} \mathrm{C}$, while the sequence with concentration $3 \% \mathrm{w} / \mathrm{v}$ and $40{ }^{\circ} \mathrm{C}$ shows the same non-monotonic trend already observed at $25{ }^{\circ} \mathrm{C}$. The non monotonic hight trends observed for $3 \%$ alginate concentrations (both at $25{ }^{\circ} \mathrm{C}$ and $40{ }^{\circ} \mathrm{C}$ ) is produced by the presence of irregularities in 
the thread shapes as the one represented in Fig. 4 . These irregularities manifest for large nozzle velocities and $3 \%$ alginate concentrations are explained by viscous effect (see the discussion below) and determine also the behaviour of the coverage ratio. The coverage ratio, reported for the four cases in Figure 2(b), allows a similar classification, showing a maximum around $v_{\text {nozzle }} \approx 0.8 v_{\text {ink }}$ for solutions with $3 \%$ concentration. Then, the coverage ratio decreases at higher nozzle velocity values due to the irregular shape of the deposited ink as reported in the ink density map of Fig. 2 (panel a). The distinct irregular signature in the thread decreases the coverage ration at $v_{\text {nozzle }}=1.75 v_{\text {ink }}$.

In Fig. 4 the fluid viscosity of the mixture is reported for the case $3 \% \mathrm{w} / \mathrm{v}$ at $25{ }^{\circ} \mathrm{C}$ after the ink is deposited. As a first, we observe in Fig. 4 overall a low viscosity in the extruded fluid part which is the result the shear rate enforced among the moving nozzle and the substrate. In all the simulations, we observe a detachment point of the ink from the substrate. In particular, the shearing force produces the detachment point just after the ink reaches the substrate. Later, the detachment point remains visible as an irregular blob in the thread (see Fig. 4). Then, the tread reabsorbs the blob under the action of the capillary pressure. Hence, the rheological behaviour of non-Newtonian inks play a central role in this process. In particular, the relation between shear-rate and the viscosity tunes the magnitude of the transmitted nozzle movements to the deposited ink, biasing both the thread shape and the quality of the final products. Further, it is observed the presence of a low viscosity close to the wall of the nozzle(see Fig. 4), which is consistent with the Poiseuille flow as a consequence of the larger velocity gradient, $\partial v / \partial x$, close to the no-slip boundaries. On the other hand, the viscosity profile shows a high peak in the middle of the nozzle (corresponding to the lowest velocity gradient point), which can be relevant for the cell viability in bio-inks. Indeed, in the context of cell culture applications, shear stress is essential to control cell viability, which may be compromised by the impact force generated by high gradient in viscosity within the nozzle channel $53 \mid 54$.

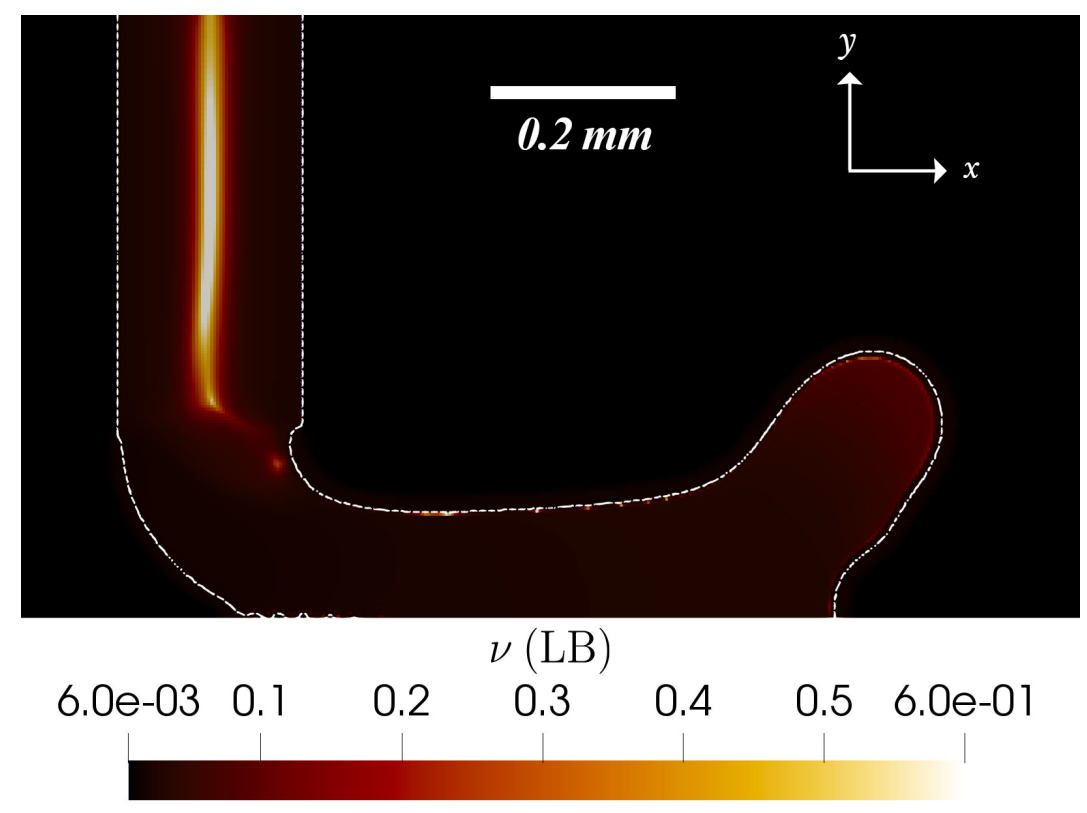

Figure 4. Kinematic viscosity map of the mixture, $\nu$, for the case $3 \% \mathrm{w} / \mathrm{v}$ at $25{ }^{\circ} \mathrm{C}$, in LB units, after the ink is deposited. The dashed white line highlights the fluid interface. The ink on the right side features higher viscosity than in the contact line with the substrate.

The $P O I_{i}^{\text {norm }}$ values, for thread heights less than two times the nozzle diameter, are assessed and shown in Fig 5 . Increasing the alginate concentration results in higher $P O I_{i}^{n o r m}$ values, which is mainly due to the higher coverage ratios $A_{i} / A_{r}$ alongside with smaller variation in the ink pressure. In particular, for $3 \% \mathrm{w} / \mathrm{v}$ concentrations, the ratio $\Delta p_{m} / p_{0}$ is found in the range from 0.1 to 0.2 for slow nozzle velocity $v_{\text {nozzle }}<0.8 v_{\text {ink }}$. On the contrary, $\Delta p_{m} / \Delta p_{0}$ is always larger than 0.4 for $1 \%$ and $2 \% \mathrm{w} / \mathrm{v}$ and for all the nozzle velocities, providing lower $P O I_{i}^{\text {norm }}$ values. Thus, the $P O I_{i}^{\text {norm }}$ shows a peak at moderate nozzle speed in the range $0.6-0.8 v_{\text {ink }}$ at high alginate concentration $3 \%$ $\mathrm{w} / \mathrm{v}$, due to the simultaneous concourse of high coverage ratios and small ink pressure variations.

Finally, in some cases, small porosity was noted in the ink fluid (see the upper panel of Fig. 2). In order to address the question, the POI values were reconsidered, taking into account the porosity. Indeed, since $A_{i}$ represents the area covered by the ink, the area decreases as the porosity increases in the fluid, whenever the porous are excluded in the $A_{i}$ assessment. The POI results are practically unaffected by this new definition, with $A_{i}$ values always changing less than $1 \%$. Consequently, the porosity in the trend-line does not bias the features of printed material in the present 


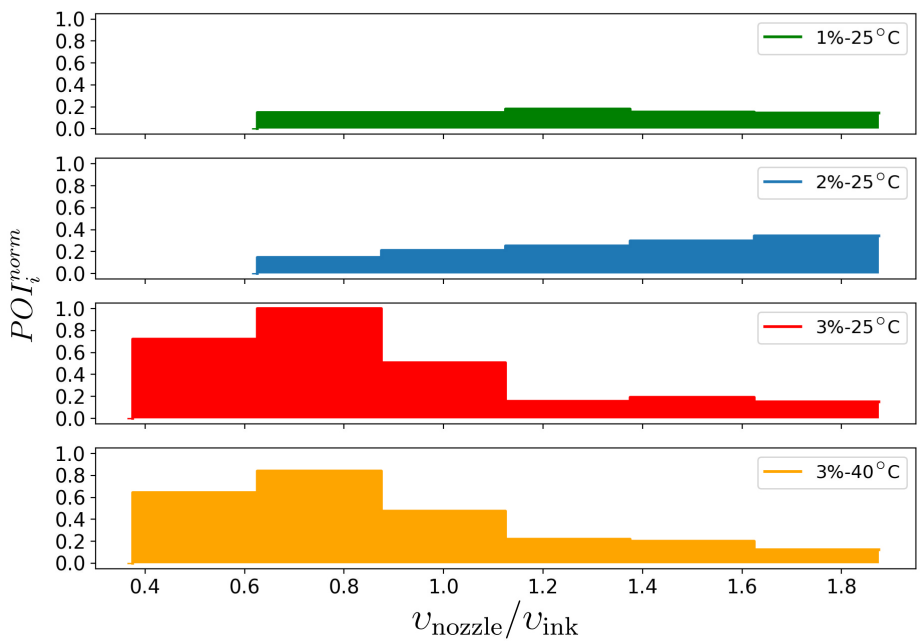

Figure 5. Parameter optimization index, reported for thread heights less than two times the nozzle diameter, for different alginate concentrations and temperatures.

simulations, and its effect can be reasonably neglected.

\section{CONCLUSIONS}

Summarising, we have introduced a multi-component model of non-Newtonian inks through a regularised version of the colour gradient LB model and used it to simulate the printing process as a function of a number of design parameters. The model allows to calculate the shear stresses during the printing process of non-Newtonian inks, directly accessible by simulations, that is very important to control the cell viability in bio-inks. The print accuracy

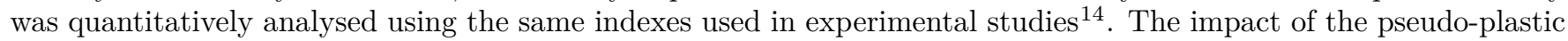
rheology on the printing accuracy was investigated for a set of solutions at different alginate concentration. Systematic investigations of processes are enabled on a broad viscosity range, providing a useful tool to probe the dynamics of the forces acting inside and on the ink during additive manufacturing. In real systems, shear thinning fluids are usually employed in order to favour the throughput of the device (small viscosity at high shear rates) and to obtain a stable and regular thread at the end of deposition (high viscosity at small shear rates). However the accuracy of the deposited threads is deteriorated for high viscosity alginate concentration at high print speed since these composites favour the transmission of the inertia of the fluid impacting the substrate which produce irregularities in the threads.

\section{DATA AVAILABILITY}

Data available on request from the authors.

\section{ACKNOWLEDGMENTS}

The research leading to these results has received funding from MIUR under the project "3D-Phys" (PRIN 2017PHRM8X), and from the European Research Council under the European Union's Horizon 2020 Framework Programme (No. FP/2014- 2020)/ERC Grant Agreement No. 739964 ("COPMAT"). We acknowledge the CINECA project DI3PRI under the ISCRA initiative, for the availability of high performance computing resources and support.

\section{REFERENCES}

${ }^{1}$ R. L. Truby and J. A. Lewis, "Printing soft matter in three dimensions," Nature 540, 371-378 (2016). 
${ }^{2}$ T. D. Ngo, A. Kashani, G. Imbalzano, K. T. Nguyen, and D. Hui, "Additive manufacturing (3d printing): A review of materials, methods, applications and challenges," Composites Part B: Engineering 143, 172-196 (2018).

${ }^{3}$ R. D. Farahani, M. Dubé, and D. Therriault, "Three-dimensional printing of multifunctional nanocomposites: manufacturing techniques and applications," Advanced Materials 28, 5794-5821 (2016).

${ }^{4}$ E. Axpe and M. L. Oyen, "Applications of alginate-based bioinks in 3d bioprinting," International journal of molecular sciences 17, 1976 (2016).

${ }^{5}$ B. Utela, D. Storti, R. Anderson, and M. Ganter, "A review of process development steps for new material systems in three dimensional printing (3dp)," Journal of Manufacturing Processes 10, 96-104 (2008).

${ }^{6}$ M. Lauricella, S. Succi, E. Zussman, D. Pisignano, and A. L. Yarin, "Models of polymer solutions in electrified jets and solution blowing," Reviews of Modern Physics 92, 035004 (2020).

${ }^{7}$ A. M. Gañán-Calvo, J. M. López-Herrera, M. A. Herrada, A. Ramos, and J. M. Montanero, "Review on the physics of electrospray: from electrokinetics to the operating conditions of single and coaxial taylor cone-jets, and ac electrospray," Journal of Aerosol Science 125, 32-56 (2018).

${ }^{8}$ S. Succi, The lattice Boltzmann equation: for complex states of flowing matter (Oxford University Press, 2018).

${ }^{9}$ T. Krüger, H. Kusumaatmaja, A. Kuzmin, O. Shardt, G. Silva, and E. M. Viggen, "The lattice boltzmann method," Springer International Publishing 10, 978-3 (2017).

${ }^{10}$ H. Huang, M. Sukop, and X. Lu, Multiphase lattice Boltzmann methods: Theory and application (John Wiley \& Sons, 2015).

${ }^{11}$ R. Benzi, S. Succi, and M. Vergassola, "the lattice boltzmann equation: theory and applications"," Physics Reports 222, 145-197 (1992).

${ }^{12}$ M. M. Cross, "Rheology of non-newtonian fluids: a new flow equation for pseudoplastic systems," Journal of colloid science 20, 417-437 (1965).

${ }^{13}$ J. Latt and B. Chopard, "Lattice boltzmann method with regularized pre-collision distribution functions," Mathematics and Computers in Simulation 72, 165-168 (2006).

${ }^{14}$ B. Webb and B. J. Doyle, "Parameter optimization for 3d bioprinting of hydrogels," Bioprinting 8, 8-12 (2017).

${ }^{15}$ A. Montessori, M. Lauricella, M. La Rocca, S. Succi, E. Stolovicki, R. Ziblat, and D. Weitz, "Regularized lattice boltzmann multicomponent models for low capillary and reynolds microfluidics flows," Computers \& Fluids 167, 33-39 (2018).

${ }^{16}$ S. Leclaire, A. Parmigiani, O. Malaspinas, B. Chopard, and J. Latt, "Generalized three-dimensional lattice boltzmann color-gradient method for immiscible two-phase pore-scale imbibition and drainage in porous media," Physical Review E 95, 033306 (2017).

${ }^{17}$ S. Leclaire, M. Reggio, and J.-Y. Trépanier, "Progress and investigation on lattice boltzmann modeling of multiple immiscible fluids or components with variable density and viscosity ratios," Journal of Computational Physics 246, 318-342 (2013).

${ }^{18}$ S. Leclaire, A. Parmigiani, B. Chopard, and J. Latt, "Three-dimensional lattice boltzmann method benchmarks between color-gradient and pseudo-potential immiscible multi-component models," International Journal of Modern Physics C 28, 1750085 (2017).

${ }^{19}$ Z. Wen, Q. Li, Y. Yu, and K. H. Luo, "Improved three-dimensional color-gradient lattice boltzmann model for immiscible two-phase flows," Physical Review E 100, 023301 (2019).

${ }^{20}$ S. Saito, Y. Abe, and K. Koyama, "Lattice boltzmann modeling and simulation of liquid jet breakup," Physical Review E 96, 013317 (2017).

${ }^{21}$ H. Liu, A. J. Valocchi, and Q. Kang, "Three-dimensional lattice boltzmann model for immiscible two-phase flow simulations," Physical Review E 85, 046309 (2012).

${ }^{22}$ T. Reis and T. Phillips, "Lattice boltzmann model for simulating immiscible two-phase flows," Journal of Physics A: Mathematical and Theoretical 40, 4033 (2007).

${ }^{23}$ M. Latva-Kokko and D. H. Rothman, "Static contact angle in lattice boltzmann models of immiscible fluids," Physical Review E 72 046701 (2005).

${ }^{24}$ T. Akai, B. Bijeljic, and M. J. Blunt, "Wetting boundary condition for the color-gradient lattice boltzmann method: Validation with analytical and experimental data," Advances in water resources 116, 56-66 (2018).

${ }^{25}$ S. Leclaire, K. Abahri, R. Belarbi, and R. Bennacer, "Modeling of static contact angles with curved boundaries using a multiphase lattice boltzmann method with variable density and viscosity ratios," International Journal for Numerical Methods in Fluids 82, 451-470 (2016).

${ }^{26}$ R. Zhang, X. Shan, and H. Chen, "Efficient kinetic method for fluid simulation beyond the navier-stokes equation," Physical Review E 74, $046703(2006)$

${ }^{27}$ C. G. Coreixas, High-order extension of the recursive regularized lattice Boltzmann method, Ph.D. thesis (2018).

${ }^{28}$ A. Montessori, G. Falcucci, P. Prestininzi, M. La Rocca, and S. Succi, "Regularized lattice bhatnagar-gross-krook model for two-and three-dimensional cavity flow simulations," Physical Review E 89, 053317 (2014).

${ }^{29} \mathrm{~J}$. Latt, Hydrodynamic limit of lattice Boltzmann equations, Ph.D. thesis, University of Geneva (2007).

${ }^{30}$ M. Lauricella, S. Melchionna, A. Montessori, D. Pisignano, G. Pontrelli, and S. Succi, "Entropic lattice boltzmann model for charged leaky dielectric multiphase fluids in electrified jets," Physical Review E 97, 033308 (2018).

${ }^{31}$ G. Pontrelli, S. Ubertini, and S. Succi, "The unstructured lattice boltzmann method for non-newtonian flows," Journal of Statistical Mechanics: Theory and Experiment 2009, P06005 (2009).

${ }^{32}$ S. Gabbanelli, G. Drazer, and J. Koplik, "Lattice boltzmann method for non-newtonian (power-law) fluids," Physical review E 72, $046312(2005)$.

${ }^{33}$ E. Aharonov and D. H. Rothman, "Non-newtonian flow (through porous media): A lattice-boltzmann method," Geophysical Research Letters 20, 679-682 (1993).

${ }^{34}$ O. Malaspinas, G. Courbebaisse, and M. Deville, "Simulation of generalized newtonian fluids with the lattice boltzmann method," International Journal of Modern Physics C 18, 1939-1949 (2007).

${ }^{35}$ R. Ouared and B. Chopard, "Lattice boltzmann simulations of blood flow: non-newtonian rheology and clotting processes," Journal of statistical physics 121, 209-221 (2005).

${ }^{36}$ B. S. Roopa and S. Bhattacharya, "Characterisation and modelling of time-independent and time-dependent flow behaviour of sodium alginate dispersions," International journal of food science \& technology 44, 2583-2589 (2009).

${ }^{37}$ A. J. Ladd, "Numerical simulations of particulate suspensions via a discretized boltzmann equation. part 1. theoretical foundation," Journal of fluid mechanics 271, 285-309 (1994).

${ }^{38}$ F. Jansen and J. Harting, "From bijels to pickering emulsions: A lattice boltzmann study," Physical Review E 83, 046707 (2011). 
${ }^{39}$ A. Ladd and R. Verberg, "Lattice-boltzmann simulations of particle-fluid suspensions," Journal of statistical physics 104, 1191-1251 (2001).

${ }^{40}$ C. K. Aidun, Y. Lu, and E.-J. Ding, "Direct analysis of particulate suspensions with inertia using the discrete boltzmann equation," Journal of Fluid Mechanics 373, 287-311 (1998).

${ }^{41}$ I. Ginzburg, "Generic boundary conditions for lattice boltzmann models and their application to advection and anisotropic dispersion equations," Advances in Water Resources 28, 1196-1216 (2005).

${ }^{42}$ A. Kupershtokh, D. Medvedev, and D. Karpov, "On equations of state in a lattice boltzmann method," Computers \& Mathematics with Applications 58, 965-974 (2009).

${ }^{43} \mathrm{M}$. Sarker and X. Chen, "Modeling the flow behavior and flow rate of medium viscosity alginate for scaffold fabrication with a threedimensional bioplotter," Journal of Manufacturing Science and Engineering 139 (2017).

${ }^{44}$ E. Ilhan, S. Cesur, E. Guler, F. Topal, D. Albayrak, M. M. Guncu, M. E. Cam, T. Taskin, H. T. Sasmazel, B. Aksu, F. N. Oktar, and O. Gunduz, "Development of satureja cuneifolia-loaded sodium alginate/polyethylene glycol scaffolds produced by 3d-printing technology as a diabetic wound dressing material," International Journal of Biological Macromolecules 161, 1040-1054 (2020).

${ }^{45}$ P. Del Gaudio, P. Colombo, G. Colombo, P. Russo, and F. Sonvico, "Mechanisms of formation and disintegration of alginate beads obtained by prilling," International journal of pharmaceutics 302, 1-9 (2005).

${ }^{46}$ Y. He, F. Yang, H. M. Zhao, Q. Gao, B. Xia, and J. Z. Fub, "Research on the printability of hydrogels in 3d bioprinting," Scientific Reports 6, 29977 (2016).

${ }^{47}$ J. Ventrici de Souza, Y. Liu, S. Wang, P. Dörig, T. L. Kuhl, J. Frommer, and G.-y. Liu, "Three-dimensional nanoprinting via direct delivery," The Journal of Physical Chemistry B 122, 956-962 (2018).

${ }^{48}$ D. Ye, Y. Ding, Y. Duan, J. Su, Z. Yin, and Y. A. Huang, "Large-scale direct-writing of aligned nanofibers for flexible electronics," Small 14, $1703521(2018)$.

${ }^{49}$ M. Wei, F. Zhang, W. Wang, P. Alexandridis, C. Zhou, and G. Wu, "3d direct writing fabrication of electrodes for electrochemical storage devices," Journal of Power Sources 354, 134-147 (2017).

${ }^{50}$ Y. Jin, W. Chai, and Y. Huang, "Printability study of hydrogel solution extrusion in nanoclay yield-stress bath during printing-thengelation biofabrication," Materials Science and Engineering: C 80, 313-325 (2017).

${ }^{51}$ Z. Zhang, Y. Jin, J. Yin, C. Xu, R. Xiong, K. Christensen, B. R. Ringeisen, D. B. Chrisey, and Y. Huang, "Evaluation of bioink printability for bioprinting applications," Applied Physics Reviews 5, 041304 (2018).

52 J.-F. Agassant, F. Pigeonneau, L. Sardo, and M. Vincent, "Flow analysis of the polymer spreading during extrusion additive manufacturing," Additive Manufacturing 29, 100794 (2019).

${ }^{53}$ J. Shi, B. Wu, S. Li, J. Song, B. Song, and W. F. Lu, "Shear stress analysis and its effects on cell viability and cell proliferation in drop-on-demand bioprinting," Biomedical Physics \& Engineering Express 4, 045028 (2018).

${ }^{54}$ F. Lee, C. Iliescu, F. Yu, and H. Yu, "Constrained spheroids/organoids in perfusion culture," in Methods in cell biology, Vol. 146 (Elsevier, 2018) pp. 43-65. 